\title{
ESPACIOS DE LIBERTAD: MUJERES, VIOLENCIA DOMÉSTICA Y RESISTENCIA ${ }^{1}$
}

\section{Diana Valle Ferrer ${ }^{2}$}

Este libro recorre la trayectoria de muchos años de análisis y reflexión sobre la violencia doméstica, sus raíces, marcos teóricos y debates, así como las experiencias de mujeres victimas/ sobrevivientes, sus luchas y su resistencia. ¿Cómo sobreviven? ¿Cómo resisten? ¿Cómo tienen fuerzas para seguir luchando ante la adversidad? Estas son las preguntas medulares que el texto aborda.

Este trabajo asume una postura feminista que reconoce la opresión contra las mujeres desde una perspectiva teórica de intersección entre los sistemas de opresión, de género, clase, etnia, raza y sexualidad. Se analiza la multidimensionalidad de la violencia contra las mujeres en las relaciones de intimidad, y las dinámicas de poder, control y resistencia. La perspectiva feminista y de género que guía este trabajo se enfoca en la resistencia de las mujeres en su vida personal, familiar y social. Además, propone un modelo de empoderamiento de las mujeres ante la opresión y la violencia.

En el análisis se consideran las dimensiones personales, interpersonales y sociopolíticas, el proceso complejo del cual una mujer entra y sale de una relación violenta, así como el lugar socio histórico donde están situadas y las múltiples opresiones que enfrentan. Uno de los objetivos de este libro es enfatizar lo imprescindible que es entender la complejidad de la violencia doméstica y reconocer que las mujeres constantemente las mujeres desafían las definiciones tradicionales de lo que es enfrentar una relación abusiva.

El libro consta de seis capítulos, además de la introducción y apéndices. El primer capítulo es un análisis de la literatura del área deviolencia contra las mujeres en la familia y la sociedad, que

${ }^{1}$ Espacio Editorial, Buenos Aires, Argentina, 2011. ISBN 978-950-802-

${ }^{2}$ Catedrática Escuela Graduada de Trabajo Social Beatriz Lassalle, Universidad de Puerto Rico en Río Piedras. 
incluyelos debates sobre definiciones, incidencia y prevalencia. La autora clarifica las diferencias y semejanzas entre conceptos tales como violencia familiar, violencia conyugal, violencia doméstica, violencia de género y violencia contra las mujeres en las relaciones íntimas o de pareja, y examina los debates sobre la simetría o asimetría de la violencia en las relaciones de pareja y los diferentes tipos de violencia. Además, se evalúa las estadísticas de incidencia y prevalencia en Puerto Rico, Estados Unidos e internacionales.

En el segundo capítulo, la autora analiza los diferentes marcos y enfoques teóricos que se han utilizado para entender y explicar la violencia contra las mujeres en la sociedad, la familia y en la relación de pareja. Comienza con las teorías sobre la opresión contra las mujeres y luego examina las explicaciones de diferentes disciplinas sobre la violencia contra las mujeres en la relación de pareja. Valle Ferrer utiliza una perspectiva feminista en su análisis de la violencia. En el tercer capitulo, se presentan algunas teorías feministas sobre la resistencia de las mujeres ante la opresión y los dictados culturales patriarcales. Se revisa la literatura sobre investigaciones históricas, sociales y psicológicas que intentan contestar cómo las mujeres resisten, responden y enfrentan la opresión y el maltrato en la sociedad, la familia y en las relaciones de intimidad.

En el cuarto capítulo, la autora explica cómo desde su perspectiva y la de mujeres sobrevivientes, acontece la violencia doméstica: cómo se inicia, se instaura, se mantiene por un periodo de tiempo y, finalmente, se rompe el control que ejerce el hombre sobre la mujer. Valle Ferrer identifica tres etapas y dos momentos cruciales en el proceso de entrampamiento y liberación de una relación de maltrato. Además, se discuten las estrategias del poder y control, así como las estrategias de resistencia de las mujeres ante la violencia. En el quinto capitulo, Valle Ferrer propone los lineamientos generales de un modelo de empoderamiento para trabajar con mujeres sobrevivientes de violencia partiendo de la resistencia y fortalezas de las mujeres. El modelo esta basado en teorias de estrés y enfrentamiento, conceptos de poder y resistencia, una perspectiva feminista sobre la opresión y la violencia contra las mujeres y teorías de empoderamiento. El modelo plantea estrategias y técnicas de intervención para apoyar a las mujeres en su proceso de empoderamiento.

En el sexto y último capítulo, la autora pasa revista sobre muchas de las instancias que provocaron interés en el área de resistencia de las mujeres ante la opresión y la violencia. Valle Ferrer también hace una reflexión sobre los logros y obstáculos que el movimiento feminista ha confrontado en ubicar la violencia contra las mujeres como un asunto importante en la discusión pública y de las políticas sociales en Puerto Rico. 